\title{
A mean field model of dynamic and post-dynamic recrystallization predicting kinetics, grain size and flow stress
}

\author{
O. Beltran ${ }^{\mathrm{a}}$, K. Huang ${ }^{\mathrm{b}, *}$, R.E. Logé ${ }^{\mathrm{a}, \mathrm{b}}$ \\ a Mines ParisTech, CEMEF-Centre for Material Forming, CNRS UMR 7635, BP 207, 1 Rue Claude Daunesse, 06904 Sophia Antipolis Cedex, France \\ ${ }^{\mathrm{b}}$ Thermomechanical Metallurgy Laboratory - PX Group Chair, Ecole Polytechnique Fédérale de Lausanne (EPFL), CH-2002 Neuchâtel, Switzerland
}

\section{A R T I C L E I N F O}

\section{Article history:}

Received 5 January 2015

Received in revised form 20 February 2015

Accepted 23 February 2015

\section{Keywords:}

Modeling

Dynamic recrystallization

Post dynamic recrystallization

Grain growth

Nucleation

304L steel

\begin{abstract}
A B S T R A C T
A physically-based two-site mean field model has been developed to describe the microstructural evolution due to recrystallization during and after deformation. The model has been applied to predict the recrystallized fraction, recrystallized grain size, and flow stress of 304L austenitic stainless steel during discontinuous dynamic recrystallization (DDRX), post-dynamic recrystallization (PDRX) and grain growth (GG). The model parameters vary with temperature and strain rate but do not depend on grain size. In PDRX and GG regime, the parameters only depend on temperature. The model responds well to conditions with different temperatures, strain rates, strains and/or annealing times. Particular attention is paid to the occurrence of two-stage growth in the recrystallized grain size plots when PDRX occurs. There is a good quantitative agreement between model predictions and experimental results obtained in the different recrystallization regimes, opening the possibility of modeling multi-pass operations compatible with industrial applications.
\end{abstract}

(c) 2015 Elsevier B.V. All rights reserved.

\section{Introduction}

Recrystallization is an important phenomenon that causes microstructural changes in deformed materials, thereby affecting its properties. Many metallic components are produced from large castings, which are then further deformed by forging, extrusion, rolling, etc., into an intermediate or final product. Three different recrystallization behaviors are usually observed during these processes, namely, dynamic, post-dynamic and static recrystallization. The recrystallization regime depends on whether it occurs during hot deformation or between deformation intervals.

In static recrystallization (SRX), the deformation is interrupted before reaching the critical strain for dynamic recrystallization (DRX), $\varepsilon_{c r}^{D R X}$. During this recrystallization regime, both nucleation and growth of new grains take place during annealing periods between intervals of hot deformation. On the other hand, these processes can also be observed during deformation once $\varepsilon_{c r}^{D R X}$ is reached, leading to dynamic recrystallization. Post-dynamic recrystallization (PDRX) refers to the growth of dynamically formed nuclei once deformation (greater than $\varepsilon_{c r}^{D R X}$ ) has come to an end. Grain growth (GG) is defined as the uniform coarsening

\footnotetext{
* Corresponding author. Tel.: +41216954472.

E-mail address: ke.huang@epfl.ch (K. Huang).
}

of a material with a minimal dislocation content at a high temperature [1].

Although extensive efforts have been put on a wide range of materials, recrystallization during and following deformation is still not fully understood [2]. Experimentally, the softening fraction [3] during annealing of dynamically recrystallized microstructures was often used to differentiate recrystallization regimes, due to the fact that it is difficult to distinguish SRX and PDRX [4]. Researchers at McGill University were probably the first ones who investigated the post-dynamic softening. They proposed a mechanism involving three different restoration processes including recovery, PDRX, and SRX, based on metallographic examination on polycrystalline copper [5-7]. Later, Sakai et al. [8,9] performed further research on the subject using a nickel alloy and austenitic steels, providing a detailed description of the above restoration processes. The observed fractional softening is, however, a combined effect of recovery, recrystallization and (where applicable) precipitation contributions, making it hard to distinguish their separate influences. The different mechanisms acting after hot deformation have been further studied by Hodgson and co-workers [10-13], while the mechanisms of recrystallization during and after deformation have been carefully examined by Dehghan-Manshadi et al. $[14,15]$ using 304 austenitic stainless steels.

Several models have been proposed to investigate the effect of the thermomechanical parameters on the softening kinetics during 
and after deformation, however, limited studies have been reported on the microstructural evolution in terms of recrystallized volume fraction and grain size. A large group of these models are based on the Johnson-Mehl-Avrami-Kolmogorov (JMAK) equation approach [15-21]. However, the ideal JMAK behavior is rarely exhibited by real materials. Such deviation from this ideal behavior is due to the presence of recovery, non-uniform distribution of stored strain energy, non-random distribution of recrystallized nuclei, and anisotropic growth of the recrystallized nuclei [22]. For this category of models, experimental validation is often lacking, and good agreement between numerical and experimental data in terms of grain size is rare. Beyond the JMAK model, the evolution of dislocation density populations can be discretely followed through the entire deformation process, e.g. by Roucoules et al. [23], based on the Sandstrom and Lagneborg approach [24,25]. However, in [23], the recrystallized grain size, which is of interest in most of the industrial applications, was not analyzed. It is furthermore assumed that the mechanisms which take place during dynamic and static recovery or during DRX, SRX or PDRX are the same. Kugler and Turk [26] developed a Cellular Automaton model for the simulation of multi-stage deformation and post-dynamic recrystallization for the case when DRX is initiated in the material during deformation. The model enables both quantitative and topological simulations of microstructural evolution before and during PDRX, and it allows the simulation of multi-stage deformation. However, the evolution of the recrystallized grain size was again not considered in the model. The explicit representation of microstructures includes much more information relying on the topological aspects, but when dealing with 3D microstructures with a statistical number of grains, these methods usually become computationally demanding.

Coupled models taking into account recovery, precipitation and recrystallization were also developed $[27,28]$. Numerical models [29-31] accounting for recrystallization during and after hot deformation for aluminum alloys were developed, such as the "Through Process Model" for the production processes of aluminum [32]. It should be noted that, different from austenitic stainless steels, aluminum alloys are of high stacking fault energy and recrystallization is characterized by the gradual formation and appearance of a new structure with no identifiable nucleation and growth stages.

In general, although some efforts have been reported in modeling recrystallization during multi-pass conditions for steels, the literature lacks accurate recrystallization models which are able to give quantitative agreements with experimental data (especially grain size) under multi-pass conditions. Most of these models either lack experimental validation or only provide qualitative agreement for selected deformation conditions. The connections between DRX, PDRX, SRX, and grain growth are usually oversimplified. Even though successful prediction of flow stress is usually obtained, the evolution of microstructure in terms of recrystallized fraction and grain size are mostly left unexploited. Furthermore, most of these models are not designed to operate under variable thermal and/or mechanical conditions, therefore making it difficult to use for industrial applications.

In the current work, a physically based numerical model was developed to predict the microstructural evolution during and after hot deformation at the grain scale. The framework of this model is based on a DDRX model [33] published by the current authors where the data structure is represented by a set of representative grains characterized by two state variables: grain size and dislocation density. Each representative grain is surrounded by two homogeneous equivalent media with high and low dislocation densities representing the average of recrystallized and non-recrystallized grains, respectively. The relative volume weights of the two media are related to their volume fractions, which represent a new way of introducing topological information into a DDRX model. The key phenomena like strain-hardening, recovery, nucleation of recrystallization occurring during deformation are described with traditional equations. The data structure varies with time due to nucleation and disappearance of grains until a steady-state is reached. This results in a two-site mean field model with automatic and accurate account of initial grain size effects.

In this paper, significant improvements and modifications have been made to the original DDRX model in order to account for the subsequent PDRX, making the model compatible with recrystallization after deformation. This is detailed in Section 2. The identification of model parameters is discussed in Section 3. The predictive capabilities of the model are then validated against 304L stainless steel experimental results in Section 4. The model predictions are in excellent quantitative agreement with the experimental observations under different conditions.

\section{Model description}

The model is presented as follows. Firstly, representation of the microstructure with two-site mean field media is described in Section 2.1. The general equations describing the key physical phenomena occurring during recrystallization are presented from Sections 2.2-2.4, covering the evolution of dislocation density, grain boundary migration and nucleation. Except the static recovery equation, these equations were used in our dynamic recrystallization model [33], the interested reader is referred to the original publication for more details. A more general expression of the critical dislocation for dynamic recrystallization is proposed in Section 2.5, still taking into account of the effect of dynamic recovery. The microstructure after deformation is reclassified according to the dislocation density of the grains, the details of which can be found in Section 2.6. A new formulation describing the interaction of the representative grains with the two surrounding Homogeneous Equivalent Medias (HEMs) is shown in Section 2.7, where the topological information can be incorporated due to the introduction of the two-site mean field media. Finally, the attractive features of this model and its limitation are listed in Section 2.8 .

\subsection{Microstructure representation}

The framework is based on a mean field approach [33], where each representative grain $G_{i}$, defined by two state variables (the grain diameter $D_{i}$ and the grain average dislocation density $\rho_{i}$ ), is immersed in two HEMs (see Fig. 1). A representative grain $G_{i}$ accounts for a number $N_{i}$ of identical spherical grains located at different positions in the microstructure. The neighbors of a given grain in a real recrystallizing microstructure (Fig. 1a) are replaced by two HEMs, respectively referring to the average of recrystallized (RX) and non-recrystallized (NR) grains (Fig. 1b).

The initial set of representative grains typically follows a lognormal distribution for the grain size and a Gaussian distribution for the dislocation densities. However, it has been tested that other distributions can also be used with negligible effect on the final result.

\subsection{Modeling the evolution of the dislocation density and flow stress}

The model makes use of the modified Kocks and Mecking equation [34-36] to describe the evolution of the dislocation density during deformation

$\frac{\partial \rho}{\partial \varepsilon}=K_{1}-K_{2} \rho$ 


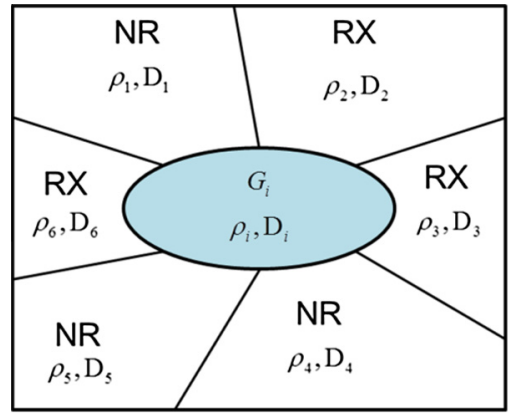

(a)

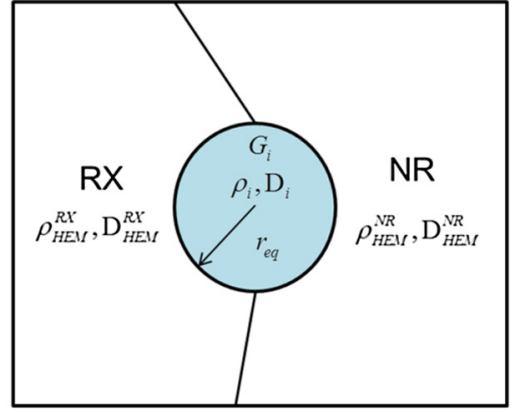

(b)

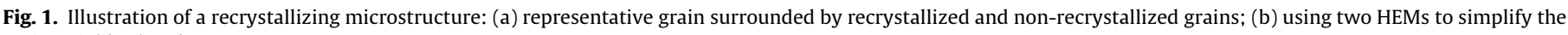
grain neighborhood.

where $K_{1}(\dot{\varepsilon})$ and $K_{2}(T, \dot{\varepsilon})$ represent, respectively, the strain hardening and recovery terms.

The flow stress $\sigma_{i}$ is calculated from its average dislocation density using the Taylor equation [37]:

$\sigma_{i}=\sigma_{0}+M \alpha \mu b \sqrt{\rho_{i}}$

where $\sigma_{0}$ a "dislocation-free" yield stress, $M$ the Taylor factor, $\alpha$ is a constant set to $0.2, \mu$ is the shear modulus, and $b$ the Burger's vector.

During post-dynamic recrystallization and subsequent grain growth, the model assumes that the dislocation density of representative grains can evolve in two different ways, namely due to static recovery and grain growth. Static recovery is only temperature dependent and it is incorporated into the model in a basic way by setting $K_{1}$ in (1) to zero and introducing the static recovery term $K_{S}$ such that,

$\frac{d \rho}{d t}=-K_{s} \rho$

Once deformation stops, a slightly deformed grain can still grow at the expense of more severely deformed ones, the area that was just swept by the moving boundary is almost dislocation free. While the total amount of dislocations existing in the growing grain is not affected by this volume change, the average dislocation density in the grain decreases due to the increase of its total volume,

$\Delta(\rho V)=0$
$\frac{\Delta \rho}{\rho}=-\frac{\Delta V}{V}$

\subsection{Modeling of grain boundary migration}

The driving force $\Delta E$ for each grain boundary migration takes into account of the stored energy due to the dislocation content [2], a capillarity term [38] related to the grain boundary energy and curvature, and the pinning effects:

$\Delta E=\tau \Delta \rho+\gamma_{b} \Delta\left(\frac{1}{r}\right)-E_{t h}$

where $\tau \approx \mu b^{2} / 2$ is the average energy per unit dislocation length, $\gamma_{b}$ the energy of the grain boundary per unit area, $r=D / 2$ is the grain radius, and $E_{\text {th }}$ is a threshold energy which accounts for pinning effects. $\Delta \rho$ and $\Delta(1 / r)$ consider the differences in dislocation density and grain size on either side of the boundary, respectively.

The grain boundary velocity evolves according to the kinetic relation [2] $v=m \Delta E$

where $m$ is the grain boundary mobility. In the present model, the mobility term is included in the $K_{3}$ parameter, given by:

$K_{3}=m \tau$

\subsection{Nucleation model}

Within certain grains where the dislocation density is greater than a critical value $\rho_{c r}$, new grains will appear due to nucleation. The nucleation rate for the $i$ th representative grain is calculated in (8)

$\dot{N}_{i, \text { nucl }}=K_{g} S_{c r} \frac{N_{i} r_{i}^{q}\left(\rho_{i}-\rho_{c r}\right)^{b_{g}}}{\sum_{\rho_{k}>\rho_{c r}} N_{k} r_{k i}^{q}\left(\rho_{k}-\rho_{c r}\right)^{b_{g}}} ; \quad K_{g}=K_{g}(T, \dot{\varepsilon})$

where $K_{\mathrm{g}}$ is a probability coefficient related to the thermo-mechanical conditions, and $S_{c r}$ the total surface area of grains with $\rho>\rho_{c r}$. Considering that the amount of activated nucleation sites per unit time increases with increasing dislocation density and grain size, the nucleation rate is further normalized by these two items. On the right side of the equation, $N_{i}$ is the number of grains associated with the $i$ th representative grain. For 304L stainless steel, nucleation during DDRX is only assumed to occur at grain boundaries through necklace-type nucleation, hence the value $q=2$ was chosen (for bulk nucleation, $q=3$ ), and $b_{g}$ is a constant with a value close to 3 [39]. All nuclei are assumed to start with a low dislocation density $\rho_{0}$.

The model assumes that no nucleation occurs during PRDX. Instead, the RX grains with dislocation densities lower than a critical dislocation density created during the last steps of DDRX are considered as RX. The recrystallization kinetics after deformation is determined by the growth of these RX grains.

\subsection{Critical dislocation density and nucleus size}

A recrystallization nucleus becomes viable when its radius reaches a critical value $r_{u}$. This corresponds to the condition when the stored energy of the material is large enough to overcome the capillary force of the nucleus (Bailey-Hirsch),

$\rho_{c r} \cdot \tau=\frac{2 \gamma_{b}}{r_{u}}$

Renaming some of the parameters in the critical dislocation density equation proposed by Roberts and Ahlblom [40], an approximate value for $\rho_{c r}^{D R X}$ can be expressed as a function of other parameters of the model: 
$\rho_{c r}^{D R X}=\left(\frac{20 \cdot K_{1} \cdot \gamma_{b} \cdot \dot{\varepsilon}}{3 \cdot K_{3} \cdot \tau}\right)^{1 / 3}$

It is observed from (10) that $\rho_{c r}^{D R X}$ is indirectly influenced by temperature and strain rate through the parameters $K_{3}$ and $K_{1}$. Temperature variation of $\rho_{c r}^{D R X}$ is mainly dictated by $K_{3}$ through the temperature dependence of the mobility parameter $m$ in (7). In general, Eq. (10) states that the critical dislocation density increases with decreasing temperature and increasing strain rate which is physically justified.

However, the critical dislocation density obtained in (10) neglects dynamic recovery. Therefore, in some cases it may not be an accurate approximation for $\rho_{c r}^{D R X}$. Dynamic recovery was taken into account to calculate the critical dislocation density in (11) (see [33]), however, it was assumed that $K_{2} \rho_{c r}^{D R X} / K_{1}$ was much smaller than 1 . In the conditions used in [32], the approximation was appropriate.

$\ln \left(1-\frac{K_{2}}{K_{1}} \rho_{c r}^{D R X}\right)=-K_{2} \frac{r_{u} \dot{\varepsilon}}{m \rho_{c r}^{D R X} \tau}$

However, when considering a wide range of thermo-mechanical conditions, $K_{2} \rho_{c r}^{D R X} / K_{1}$ may become significant. Therefore, using the expression of the critical nucleus size $r_{u}$ given by (9), $\rho_{c r}^{D R X}$ is now semi-analytically calculated as

$\rho_{c r}^{D R X}=\left[-2 \gamma_{b} \dot{\varepsilon} \frac{K_{2}}{K_{3} \tau} / \ln \left(1-\frac{K_{2}}{K_{1}} \rho_{c r}^{D R X}\right)\right]^{1 / 2}$

An initial value for $\rho_{c r}^{D R X}$ is obtained directly from (10). An iterative calculation by (12) leads to the converged value of $\rho c r^{D R X}$, used in the model.

To overcome the problem that a nucleus may shrink soon after its creation according to (9), a scaling constant $\omega$ is introduced,

$r_{u}^{D R X}=\omega \cdot \frac{2 \cdot \gamma_{b}}{\rho_{c r}^{D R X} \cdot \tau}$

where $\omega>1$. This will ensure that a created nucleus has the required driving force for growth.

\subsection{Microstructure reclassification}

If the strain applied during the deformation stage is beyond $\varepsilon_{c r}^{D R X}$, dynamic recrystallization will occur. In terms of the resulting set of representative grains during PDRX, the microstructure can be classified into four categories (Instead of three categories in [41]) due to the two-site mean field approach:

i. DRX nuclei with a dislocation density $\rho_{i}=\rho_{0}$.

ii. Growing DRX grains with $\rho_{0}<\rho_{i}<\rho_{c r}^{D R X}$.

iii. Critically work hardened DRX grains with $\rho_{i}>\rho_{c r}^{D R X}$.

iv. Critically work hardened NR grains with $\rho_{i}>\rho_{c r}^{D R X}$.

Each of these grain categories will act differently during the subsequent annealing process. For example, critically work hardened RX grains will interact with their neighboring grains in a similar way as NR grains; hence, these grains should be considered as NR grains in the following step. In order to be able to describe the different mechanisms and interactions acting in the microstructure after the interruption of deformation, a reclassification of the dynamically recrystallized grains is required. In this work, representative grains are reclassified according to their dislocation densities.

The need to reclassify the microstructure in terms of dislocation density is illustrated through an EBSD analysis. Fig. 2 shows the inverse pole figure map and the kernel average misorientation (KAM) map for a sample deformed at $1000^{\circ} \mathrm{C}$ up to a strain of 1.5 at a strain rate of $0.1 \mathrm{~s}^{-1}$. The four types of representative grains listed above can be clearly observed in Fig. 2b. Small blue grains correspond to type 1 representative grains, while fine green small grains (not the green areas inside the large deformed grains) have the characteristic description of type 2 representative grains. Type 3 grains can be observed in the top left corner in an orange-red color. NR strain-hardened grains (type 4) show a concentration of the deformation at the grain boundaries, where nucleation is observed to occur.

To reclassify the microstructure, we introduce a dislocation density threshold $\rho_{t h}$ defining the limit between RX and NR grains. The value of $\rho_{t h}$ is chosen to be the maximum dislocation density obtained when generating the initial dislocation density

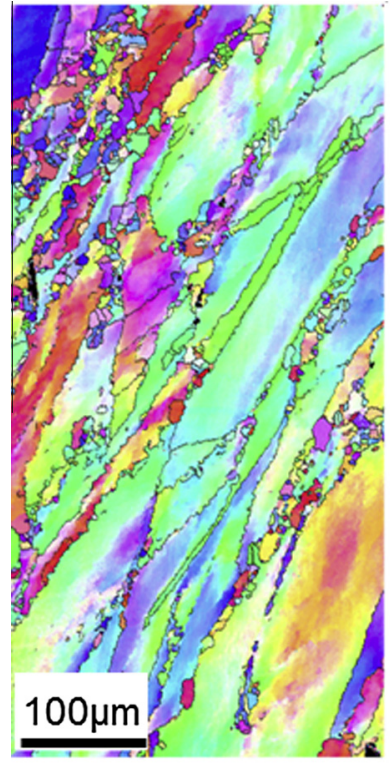

(a)

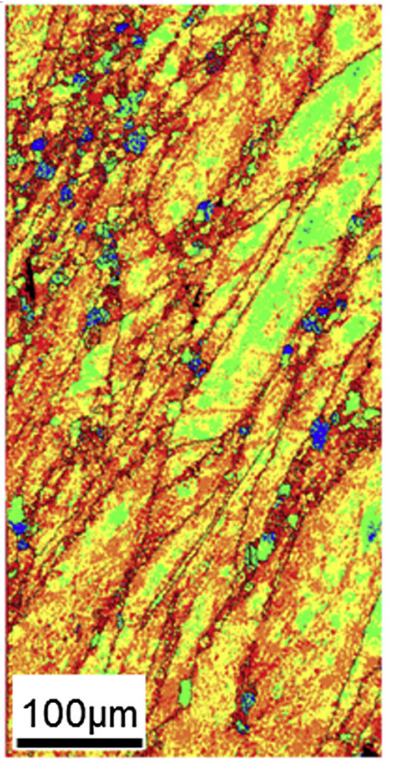

\section{Kernel average} misorientation

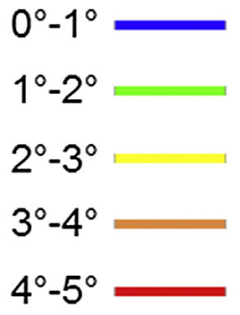

(b)

Fig. 2. Microstructure obtained after a deformation of 1.5 at a strain rate of $0.1 \mathrm{~s}^{-1}, 1000{ }^{\circ} \mathrm{C}$. (a) Inverse pole figure map; (b) Kernel average misorientation map. 


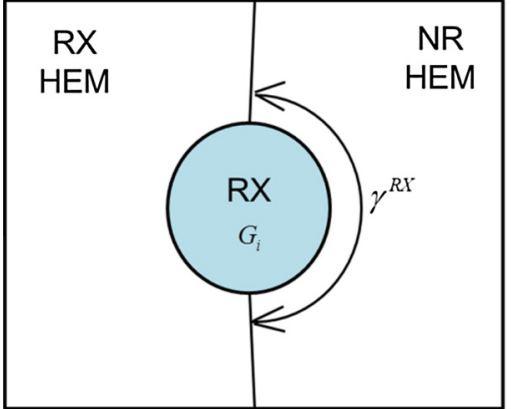

(a)

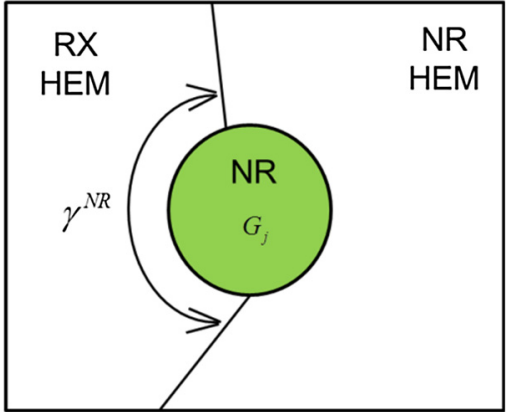

(b)

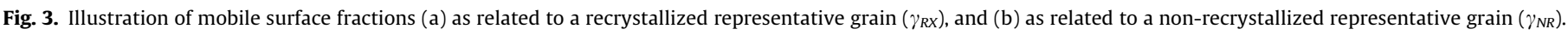

distribution (Gaussian distribution) for the starting (annealed) microstructure. When there is a change in the recrystallization regime, the representative grains with a dislocation density higher than $\rho_{\text {th }}$ are classified as NR grains.

On the other hand, during PDRX the dislocation density of NR grains can be reduced due to both the effect of static recovery and grain growth, as described in Section 2.2. If during the holding time a NR representative grain reaches a dislocation density lower than $\rho_{t h}$, this grain will be automatically reclassified as RX.

The advantage of re-defining RX and NR grains as a function of their dislocation density is that the description of the microstructure is simplified: there is no need to separately identify work hardened old grains and critically work hardened recrystallized grains. Another important benefit of re-defining RX and NR grains lies in the fact that representative grains exhibiting similar behavior are grouped accordingly, improving the approximation obtained through the use of 2 HEMs.

\subsection{The interaction between representative grain and its surrounding HEMs}

In an attempt to describe the neighborhood, two different HEMs surround each representative grain were used: one with a high dislocation density and the other one with a low dislocation density. Therefore, one part of the boundary for which $\Delta E$ is high will move faster than the other part, leading to the existence of mobile surface fractions $[25,32,38]$. A schematic representation of this concept is shown in Fig. 3. The first one corresponds to the mobile fraction $\gamma_{R X}$ of a RX representative grain. The second one, $\gamma_{N R}$ is the mobile fraction of a NR representative grain.

It is convenient to use indices $i$ and $j$ to designate RX and NR grains, respectively, in the following equations. The driving forces between a representative grain and each HEM are distinguished using the notation $\Delta E_{k}^{H E M}=E_{H E M}-E_{k}-E_{t h}$, with HEM $=\mathrm{NR}$ or RX, $k=i$ or $j$, and $E=\tau \rho+\gamma_{b} / r$.

Due to the different type of interactions and driving forces occurring during the two recrystallization regimes included in the model, two different methods were used to calculate the mobile surface fractions. The first formulation is used whenever the dislocation density distribution among grains of the same category is very narrow. Therefore, it can be assumed that the driving forces acting between each representative grain with its own HEM are negligible. In this case, the evolution of the microstructure is controlled by the interaction of the representative grains with their opposite HEM. This situation arises during the modeling of DDRX, and is the reason why a simplified expression for the migration of the different interfaces can be used during this recrystallization regime. The detailed information can be found in Ref. [33].

After DDRX however, the microstructure is composed of four different types of grains as mentioned in the previous section. Grains are reclassified at the end of the deformation step in terms of their dislocation density. This results in a very diverse and heterogeneous set (both in size and dislocation density) of NR representative grains. Due to this reclassification, the interaction between NR grains and the NR HEM can no longer be considered as negligible when modeling PDRX. In order to include this effect into the model, the migration of NR-NR and RX-RX interfaces must be considered when defining the mobile surface fractions.

Similarly to the DDRX case, the calculation of the mobile surface fraction terms starts from the boundary velocity expression (7). Considering the movement of all interfaces appearing in the model, the aggregate variations of recrystallized volume (RX-RX, RX-NR) and non-recrystallized volume (NR-NR, NR-RX) are given by,

$$
\begin{aligned}
& \Delta V_{R X / N R}=\sum_{R X} \gamma^{R X} N_{i} \Delta V_{i, R X / N R}=\sum_{R X} \gamma^{R X} N_{i} 4 \pi r_{i}^{2} m \Delta E_{i}^{N R} \Delta t \\
& \Delta V_{R X / R X}=\sum_{R X}\left(1-\gamma^{R X}\right) N_{i} \Delta V_{i, R X / R X}=\sum_{R X}\left(1-\gamma^{R X}\right) N_{i} 4 \pi r_{i}^{2} m \Delta E_{i}^{R X} \Delta t
\end{aligned}
$$

and,

$$
\begin{aligned}
& \Delta V_{N R / R X}=\sum_{N R} \gamma^{N R} N_{j} \Delta V_{j, N R / R X}=\sum_{N R} \gamma^{N R} N_{j} 4 \pi r_{j}^{2} m \Delta E_{j}^{R X} \Delta t \\
& \Delta V_{N R / N R}=\sum_{N R}\left(1-\gamma^{N R}\right) N_{j} \Delta V_{j, N R / N R}=\sum_{N R}\left(1-\gamma^{N R}\right) N_{j} 4 \pi r_{j}^{2} m \Delta E_{j}^{N R} \Delta t
\end{aligned}
$$

Volume conservation implies that,

$\Delta V_{R X / N R}+\Delta V_{R X / R X}+\Delta V_{N R / R X}+\Delta V_{N R / N R}=0$

Combining (14.1-14.5) leads to,

$\gamma^{N R}=\frac{-\gamma^{R X}\left(\sum_{R X} N_{i} r_{i}^{2} \Delta E_{i}^{N R}-\sum_{R X} N_{i} r_{i}^{2} \Delta E_{i}^{R X}\right)-\left(\sum_{N R} N_{j} r_{j}^{2} \Delta E_{j}^{N R}+\sum_{R X} N_{i} r_{i}^{2} \Delta E_{i}^{R X}\right)}{\left(\sum_{N R} N_{j} r_{j}^{2} \Delta E_{j}^{R X}-\sum_{N R} N_{j} r_{j}^{2} \Delta E_{j}^{N R}\right)}$ 
or,

$\gamma^{R X}=\frac{-\gamma^{N R}\left(\sum_{N R} N_{j} r_{j}^{2} \Delta E_{j}^{R X}-\sum_{N R} N_{j} r_{j}^{2} \Delta E_{j}^{N R}\right)-\left(\sum_{N R} N_{j} r_{j}^{2} \Delta E_{j}^{N R}+\sum_{R X} N_{i} r_{i}^{2} \Delta E_{i}^{R X}\right)}{\left(\sum_{R X} N_{i} r_{i}^{2} \Delta E_{i}^{N R}-\sum_{R X} N_{i} r_{i}^{2} \Delta E_{i}^{R X}\right)}$

The overall volume change due to boundary migration alone can be expressed by,

$$
\begin{aligned}
& \Delta V_{i}=\gamma^{R X} \Delta V_{i, R X / N R}+\left(1-\gamma^{R X}\right) \Delta V_{i, R X / R X} \\
& \Delta V_{j}=\gamma^{N R} \Delta V_{j, N R / R X}+\left(1-\gamma^{N R}\right) \Delta V_{j, N R / N R}
\end{aligned}
$$

The current model assumes that no nucleation occurs during PDRX. Instead, the RX grains with low dislocation densities created during the last steps of DDRX are considered as nuclei and allowed to grow. Although still located along the grain boundaries of strain-hardened grains, these newly created nuclei are in reduced number density such that they do not form a continuous necklace along the grain boundaries. They can be thus considered as randomly distributed in space. Therefore, it can be assumed that the evolution of the $\gamma^{R X}$ mobile surface fraction can be related to the increase of the recrystallized fraction such that

$\gamma^{R X} \cong 1-X$

Similarly, considering this time the non-recrystallized volume, one finds that

$\gamma^{N R} \cong X$

Analogous to the previous model for DDRX [33], relations (19) and (20) must however satisfy the volume conservation constraints expressed by (15) and (16). To ensure volume conservation, possible strategies are to either evaluate $\gamma^{R X}$ from Eq. (19) and compute $\gamma^{N R}$ using Eq. (15) or evaluate $\gamma^{N R}$ from Eq. (20) and compute $\gamma^{R X}$ with Eq. (16). The chosen strategy goes as follows. At the beginning of the PDRX process, it is assumed that RX grains are isolated. Thus, at the very beginning $\gamma^{R X}=1$ and $\gamma^{N R}$ is calculated using Eq. (15). Once $\gamma^{N R}$ reaches a value of 1 its value is fixed and the evolution of $\gamma^{R X}$ is calculated using Eq. (16). Volume conservation is thus ensured through this switching procedure.

\subsection{Attractive features of the model and limitations}

From the above description, it can be summarized that the merits of the proposed model lie in:

(1) The ability of predicting recrystallized fraction, grain size and flow stress at the same time, with reduced computation cost.

(2) Providing topological information from the interaction of representative grains with two homogeneous equivalent media (two-site approach), which leads to an automatic and accurate account of initial grain size effects.

(3) An easy identification of model parameters from experiments, the parameters being independent from the initial grain size.

This model also has a number of limitations:

(1) The model is designed for the recrystallization during and after deformation of low stacking fault FCC materials. With only one dislocation density type to describe the internal state of the material during deformation and recrystallization, the model is not able to adequately describe the cell structure or sub-grains frequently observed for high stacking fault FCC materials like aluminum alloys. The effect of precipitation and second-phase particles is also not considered.

(2) It is based on a grain scale approach. The heterogeneity in terms of the distribution of stored energy and the spread in misorientations within each grain is not yet considered. Nucleation at heterogeneous sites, e.g. through particle stimulated nucleation (PSN), or at shear bands, is neglected.

(3) Finally, the model assumes that all grains have the same grain boundary mobility and they follow the same dislocation density evolution law. The model does not take into account the different grains crystallographic orientations and the associated differences in material properties.

\section{Parameter identification}

In order to describe the physical behavior of the material during DDRX, the model makes use of four uncertain parameters: $K_{1}$ (strain hardening), $K_{2}$ (dynamic recovery), $K_{3}$ (mobility), and $K_{g}$ (nucleation). It is assumed that these parameters are dependent on strain rate and temperature (Except for $K_{1}$ which only depends on strain rate); therefore, these parameters are identified in a point-by-point basis in the temperature/strain rate space. Once the parameters are identified in such a grid of points, intermediate values are determined using a bilinear interpolation.

The value for these parameters can be identified through purely numerical methods [33]. This approach yields multiple solutions in the form of Pareto optimal sets. However, it was found that some of the solutions failed to describe the PDRX behavior during multipass simulations. In PDRX, the evolution of the RX fraction is determined by the growth of RX grains created in the last stages of DDRX. The velocity of the grain interfaces controls this growth, which is a function of dislocation densities and the of boundary mobility. Also, since the volume of the system remains constant, the evolution of the recrystallized grain size during PDRX depends on the number of grains considered as RX. The number of grains contained in each dynamically created representative grain is determined through the parameter $K_{g}$. Thus, it was concluded that selecting parameters based only on the values obtained from DDRX data through numerical optimization was not sufficient.

One important aspect of the model is that depending on the parameters used, the mobile surface fraction terms $\gamma^{N R}$ and $\gamma^{R X}$ might present instabilities. The model reflects the physics of DDRX and PDRX as long as the following conditions are met [33],

$\frac{d \gamma^{N R}}{d X}>0$

$\frac{d \gamma^{R X}}{d X}<0$

If these conditions are not met when using a given set of parameters, it is an indication that the model is simulating an unphysical behavior and a different set of parameters must be used.

To solve the problem of multiple solutions leading to unphysical or unstable behaviors, it is required to decrease the degrees 
of freedom in the system in order to restrict the solutions to a set of physically consistent values. This was done by obtaining a first approximation for the parameters from different recrystallization regimes (where each parameter could be measured experimentally). By doing this it was possible to discard unphysical solutions and obtain a set of parameters that actually describes the physical behavior and evolution of the material.

\subsection{Strain hardening and recovery: $K_{1}$ and $K_{2}$}

The evolution of the dislocation density during deformation is controlled by the strain hardening term $K_{1}$ and the dynamic recovery term $K_{2}$. The first approximation for these values can be obtained through the stress strain curves.

Starting from the integration of (1) the description of the flow curve is given by [42],

$\sigma=\left(\sigma_{\text {sat }}^{2}-\left(\sigma_{\text {sat }}^{2}-\sigma_{0}^{2}\right) \exp \left(-K_{2} \times \varepsilon\right)\right)^{1 / 2}$

where $\sigma_{0}$ and $\sigma_{\text {sat }}$ represent the yield stress and the saturation stress respectively. $\sigma_{\text {sat }}$ depends on $K_{1}$ and $K_{2}$ according to,

$\sigma_{s a t}=M \alpha \mu b \sqrt{\frac{K_{1}}{K_{2}}}$

Since the flow stress is given by (2), it is possible to rewrite (1) in terms of the flow stress such that,

$\frac{\partial \rho}{\partial \varepsilon}=(M \alpha \mu b)^{-2} \frac{d \sigma^{2}}{d \varepsilon}=2(M \alpha \mu b)^{-2} \sigma \frac{d \sigma}{d \varepsilon}$

By combining (2) and (25), the evolution Eq. (1) can be rewritten as,

$2 \sigma \theta=K_{2} \sigma_{\text {sat }}^{2}-K_{2} \sigma^{2}$

where $\theta=d \sigma / d \varepsilon$ is the current work hardening rate. The parameter $K_{2}$ is obtained from the slope $\left(-K_{2}\right)$ of the $2 \theta \sigma$ vs. $\sigma^{2}$ from the experimental flow stress curves. $K_{1}$ is obtained from $K_{2}$ using,

$K_{1}=\frac{K_{2} \sigma_{\text {sat }}^{2}}{(M \alpha \mu b)^{-2}}$

\subsection{Grain boundary mobility: $K_{3}$}

Due to the different mechanisms occurring during DDRX, obtaining the accurate value for $K_{3}$ proves to be a difficult task. Instead, a lower bound first approximation for this parameter is obtained through grain growth experiments. It is assumed that mobility is the lowest during grain growth and increases as a function of strain rate for a given temperature.

Grain growth is directly linked to the grain boundary mobility through (7). To estimate the lower bound for $K_{3}$, the model was fitted against pure grain growth experiments. This was done by generating a dislocation free grain size distribution and monitoring the curvature driven evolution of the different representative grains. The parameter $K_{3}$ was then manually calibrated so that the model could fit the experimental data. This was done once for each temperature.

\subsection{Nucleation: $K_{g}$}

The nucleation rate parameter $K_{\mathrm{g}}$ is perhaps the most difficult parameter to measure experimentally. For this reason, this parameter is the last one to be calibrated and must be done by running a DDRX-PDRX simulation. After finding a set of parameters $\left(K_{1}, K_{2}\right.$, and $\left.K_{3}\right)$ that correctly describes the DDRX behavior, $K_{g}$ is obtained by fitting the kinetics and the grain size in the PDRX regime.
This cross-regime validation for parameter values is a method that insures and allows the model to simulate multi-pass processes using a natural set of parameters, increasing its predictive capabilities.

\section{Model validation}

The progress of the DDRX and PDRX can be directly measured from metallographic examination. However, very limited recrystallized fraction and grain size data are available in the literature. The vast majority of existing data focuses only on the softening fraction, which is the combined effect of both recrystallization and recovery if precipitation is absent. For the validation of the model, 304L austenitic stainless steel was chosen. To improve the accuracy, microstructures at different conditions were characterized by EBSD to determine the recrystallized volume fraction and grain size. Two test cases of hot deformation followed by a holding time at the same temperature were performed. This was done to analyze DRX, PDRX, and grain growth at the same time. The relative values of the fitting parameters used for 304L stainless steel are listed in Appendix B. Parameters are expressed in a dimensionless form, relative to reference values obtained at $0.01 \mathrm{~s}^{-1}$ and $1000^{\circ} \mathrm{C}$. For the PDRX/GG regime, $K_{1}$ and $K_{g}$ are both set to zero, since no deformation and nucleation of recrystallization are involved. And the dynamic recovery term $K_{2}$ is replaced by static recovery $K_{s}$. As can be seen from Fig. B-1, $K_{1}, K_{3}$ and $K_{g}$ increase with strain rate while $K_{2}$ decreases with strain rate, which are physically justified. The model was tested at different temperatures and strain rates for the different recrystallization regimes, however, only the results obtained at $1000{ }^{\circ} \mathrm{C}$ are presented in this paper. For these simulations, the initial number of representative grains was set to 50 .

\subsection{Experimental methods and thermo-mechanical treatment}

The 304L stainless steel torsion samples used had a gauge length of $15 \mathrm{~mm}$, a diameter of $6 \mathrm{~mm}$, and were machined from rolled bars. Hot torsion tests were performed according to the schematic diagram illustrated in Fig. 4. In order to achieve a homogeneous distribution of the temperature and avoid thermal fluctuations, the samples were held for $3 \mathrm{~min}$ after reaching the target temperature $T_{0}$. The initial grain size was $\sim 135 \mu \mathrm{m}$. The samples were then deformed at the temperature $T_{0}\left(1000^{\circ} \mathrm{C}\right)$ to different strains, at strain rates of 0.01 and $0.1 \mathrm{~s}^{-1}$. After the desired strain or holding time had been reached, samples were waterquenched to room temperature.

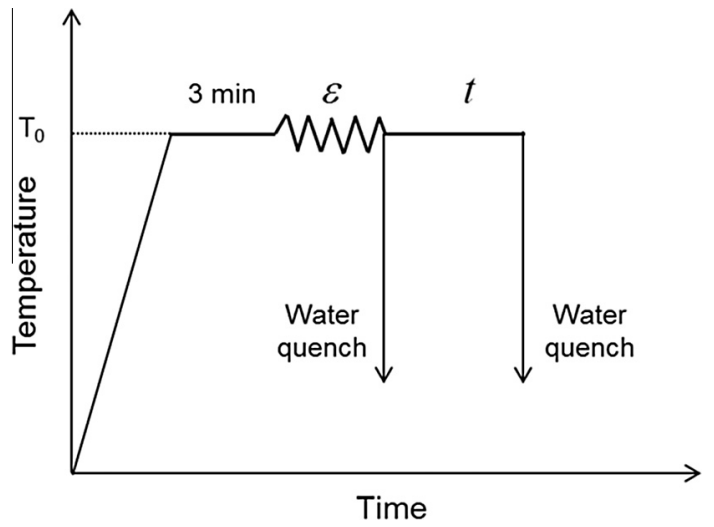

Fig. 4. Schematic diagram of torsion test for investigation of different recrystallization. 
Samples were then polished for microscopy analysis following a standard metallographic preparation: successive wet grinding starting from a 600 grit paper until 4000 grit, and a final polishing stage using colloidal silica to obtain a well finished surface. For EBSD analysis, the samples were subjected to an additional vibratory polishing for $6 \mathrm{~h}$. For optical microscopy (OM) analysis, the polished samples were etched by a chemical solution composed of $50 \mathrm{ml}$ hydrochloric acid $(\mathrm{HCl})$ and 15 drops of $\mathrm{H}_{2} \mathrm{O}_{2}(\sim 5 \mathrm{ml})$. Metallographic observations were performed on tangential sections at a depth of $\sim 100 \mu \mathrm{m}$ below the sample surface.

\subsubsection{Dynamic recrystallization}

The comparison between the experimental results and those predicted by the model for DDRX are presented in Fig. 5. The model accurately describes the evolution of the recrystallized fraction in both cases. The flow stress as a function of the strain is shown in Fig. 5a. The increase in the flow stress due to an increase in strain rate is well captured by the model. It can be observed from Fig. 5b, both numerically and experimentally, that recrystallization kinetics is faster for the test case using a strain rate of $0.01 \mathrm{~s}^{-1}$. The evolution of grain size combined with strain rate affects the shape of the flow stress curves (see Fig. 5c), and leads to a decrease in the peak stress and steady state stress.

In terms of flow stress, recrystallized fraction and grain size, there is a very good agreement between the experimental results and those predicted by the model. This good agreement shows that the identified $K$ parameters are physically consistent and describe correctly the mechanisms observed during DDRX. As mentioned in the parameter identification section, the parameters values have to be validated against PDRX. This is done by making a coupled simulation, modeling PDRX and grain growth after DDRX.

\subsubsection{Post-dynamic recrystallization}

The model was validated against two test cases during PDRX. Samples were deformed at $1000^{\circ} \mathrm{C}$ up to a strain of 1.5 , at a strain rate of $0.01 \mathrm{~s}^{-1}$ and $0.1 \mathrm{~s}^{-1}$ respectively. After deformation, samples were held at the same temperature of $1000{ }^{\circ} \mathrm{C}$ for different holding times before they were water quenched to room temperature.

To determine the RX fraction during DDRX, experimental results obtained by OM or EBSD can be directly compared to those of the model. For PDRX however, additional considerations must be taken. At the beginning and during PDRX, the model reclassifies representative grains as $\mathrm{RX}$ or $\mathrm{NR}$ in terms of their dislocation density. In order to compare the recrystallized volume fractions predicted by the model to those observed experimentally, the kernel average misorientation (KAM) maps obtained through EBSD were employed. Fig. 6 shows the microstructure after hot deformation to 1.5 at $1000{ }^{\circ} \mathrm{C}$ with a strain rate of $0.01 \mathrm{~s}^{-1}$ and with 60s' holding time before quick water quench. With the exception of a few elongated grains, Fig. 6a shows a fully recrystallized microstructure. However, after analyzing the microstructure using KAM maps (not the same observed area as OM images) obtained by EBSD, it is observed that some small grains have non-negligible stored energy. These grains were most likely created during DDRX (type 2 and 3 ) and have a similar size and shape as those which are fully recrystallized, shown in blue in Fig. 6b. The RX kinetics in PDRX is determined by the growth of grains with a dislocation density close to $\rho_{0}$ toward the deformed matrix, and the latter includes small grains created during DDRX.

The comparison between the recrystallization kinetics measured experimentally by EBSD and those obtained with the model during PDRX at $1000^{\circ} \mathrm{C}$ after a deformation of 1.5 with two strain rates of $0.01 \mathrm{~s}^{-1}$ and $0.1 \mathrm{~s}^{-1}$ is presented in Fig. 7. It should be noted that at the beginning of the PDRX process, the recrystallized fraction is close to zero, this is because only a small part of the RX

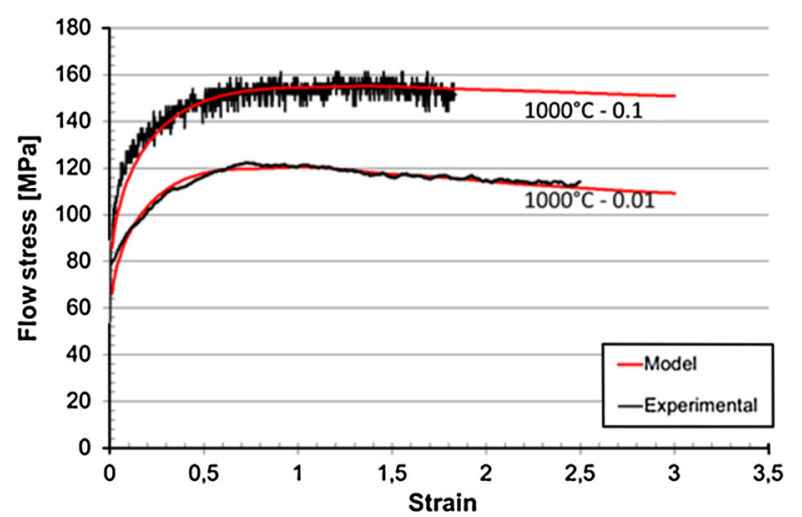

(a)

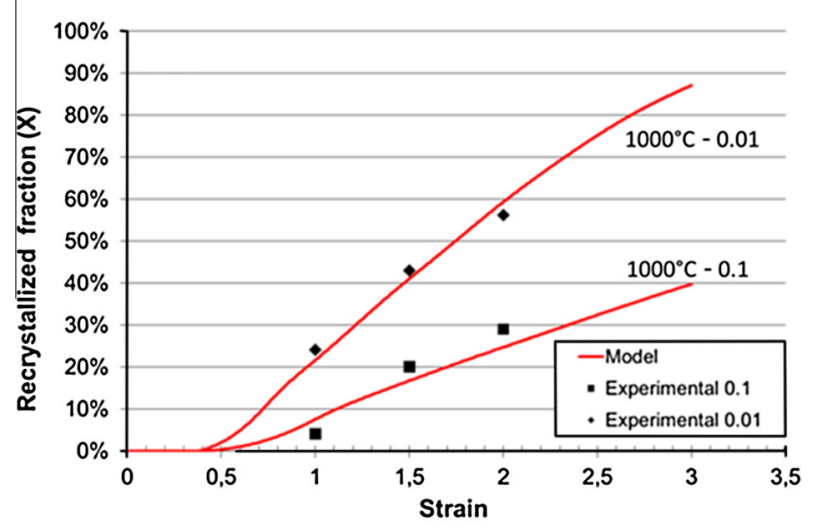

(b)

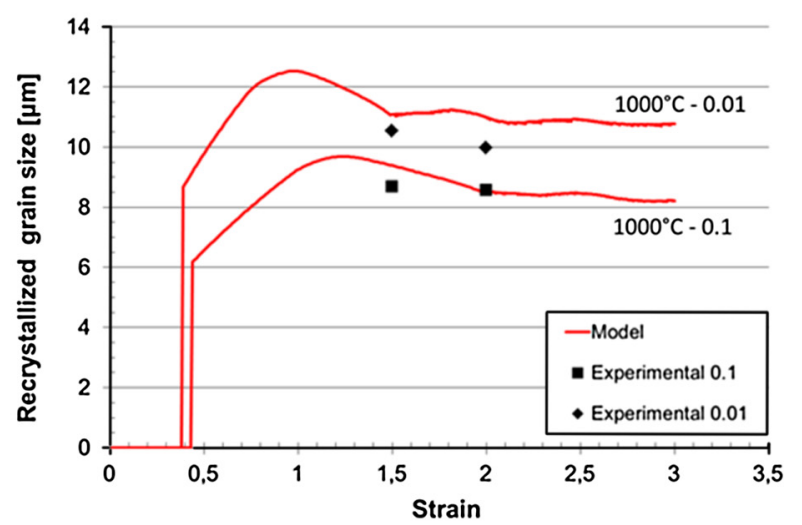

(c)

Fig. 5. Comparison between experimental and numerical results obtained with the model (DRX) (a) flow stress curves, (b) recrystallized fraction, (c) recrystallized grain size. Conditions are at $1000{ }^{\circ} \mathrm{C}, \varepsilon=0.01 \mathrm{~s}^{-1}$ and $0.1 \mathrm{~s}^{-1}$ respectively.

grains (nucleated recently) after DRX has a dislocation density less than $\rho_{t h}$, other RX grains get strained quickly. The model shows an overall good agreement for the two cases, the fact that recrystallization kinetics is faster when the strain rate is higher is also captured, but there is a slight underestimate of the recrystallized volume fraction. Since the model assumes PDRX occurs only due to the growth of dynamically created nuclei with a dislocation density lower than $\rho_{t h}$, at least part of the differences can be attributed to some type of secondary nucleation occurring in the microstructure due to SRX.

The evolution of the recrystallized grain size during PDRX for the same test cases is presented in Fig. 8. The model captures the 


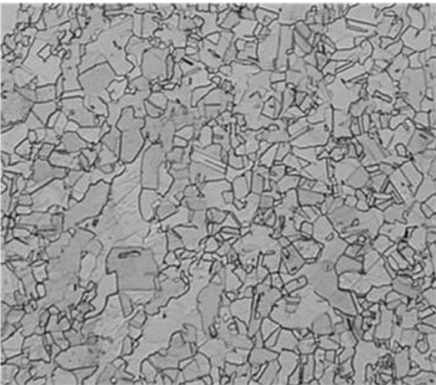

(a)

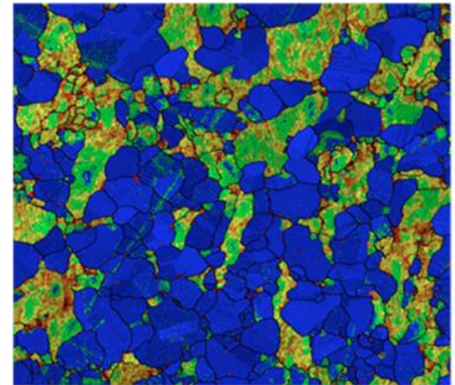

$0^{\circ}-1^{\circ}$

$1^{\circ}-2^{\circ}$

$2^{\circ}-3^{\circ}$

$3^{\circ}-4^{\circ}$

$4^{\circ}-5^{\circ}=$

\section{Kernel Average}

Misorientation

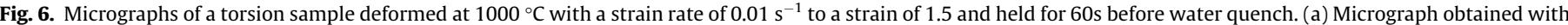

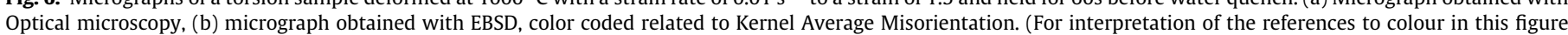
legend, the reader is referred to the web version of this article.)

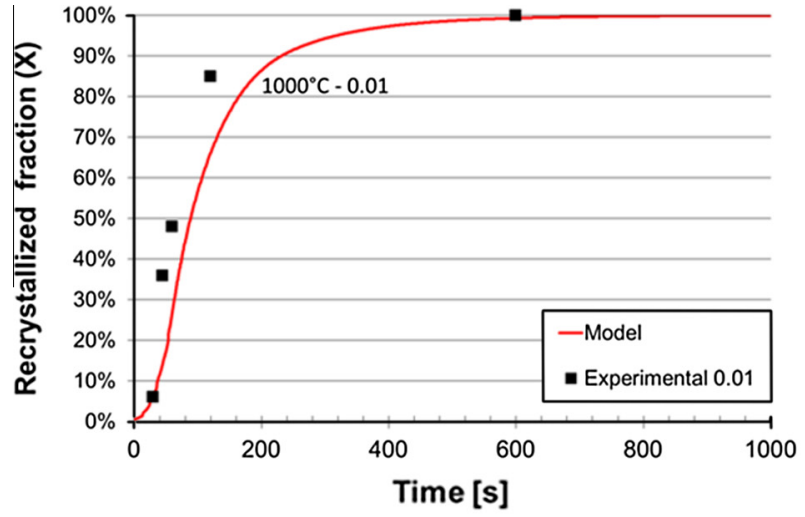

(a)

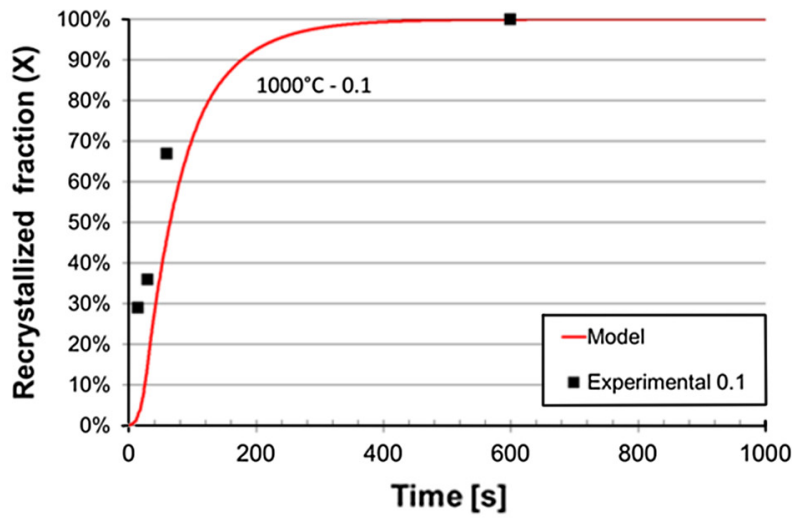

(b)

Fig. 7. PDRX kinetics after a deformation of 1.5 at $1000{ }^{\circ} \mathrm{C}$ with: (a) strain rate of $0.01 \mathrm{~s}^{-1}$, and (b) strain rate of $0.1 \mathrm{~s}^{-1}$.

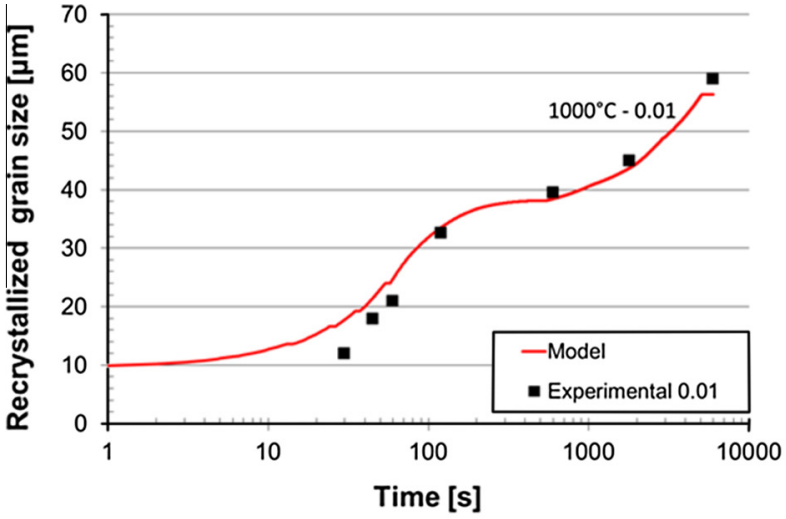

(a)

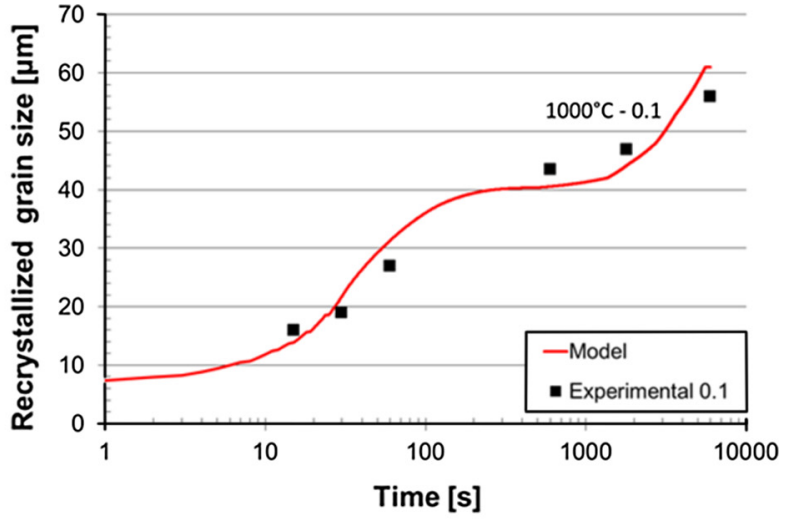

(b)

Fig. 8. Evolution of the PDRX recrystallized grain size after deformation of 1.5 at $1000{ }^{\circ} \mathrm{C}$ with: (a) strain rate of $0.01 \mathrm{~s}^{-1}$, and (b) strain rate of $0.1 \mathrm{~s}^{-1}$.

evolution accurately. Also, two distinct regimes can be observed, in accordance with existing literature $[43,44]$. At the beginning, there is a fast evolution of the grain size. This is due to the fast growth of nuclei created during the last stages of DDRX. The driving force for this growth is primarily due to the difference in dislocation density between the deformed grains and those with a dislocation density close to $\rho_{0}$. As the microstructure approaches a fully recrystallized state, the driving force and hence the grain growth rate decrease, leading to the plateau observed between 100 and 800s. Once the microstructure is fully recrystallized, normal grain growth occurs, and the driving force controlling the evolution is due to the curvature of the grains. The model is able to capture completely this two-step behavior observed experimentally.

In general, the model is able to give both qualitative and quantitative agreements between experimental results and predicted ones in terms of flow stress, recrystallized fraction and recrystallized grain size during and after hot deformation at different processing conditions. Considering its inherent simplicity, this model 
can be easily implemented into a finite element software for industrial application, since it provides a satisfactory description of different phenomena occurring during and after hot deformation. It is believed that the model has sufficient generality to be directly used for other materials with low stacking fault energy. It could also be extended to other types of materials by changing the nucleation model and/or the evolution equations of mobile surface fractions reflecting the topological description. Further efforts are being invested to improve the current model, including the analysis of the grain size distribution, the relationship between flow stress and recrystallized grain size during DDRX [45], and the strain independent characteristics of the softening associated with PDRX once a certain prestrain is attained [46]. Meanwhile, a wider range of experiments are planned to provide sufficient data for model calibration.

\section{Conclusions}

A model describing recrystallization in metallic materials capable of handling DRX, PDRX, and grain growth was developed and validated against experimental test cases for multi-pass hot deformation of 304L austenitic stainless steel. The focus of this work was to develop a numerical model able to predict microstructural evolution due to different recrystallization regimes. The key physical ingredients of this model are: a modified KocksMecking equation to follow the evolution of dislocation density, a time dependent nucleation law taking into account dynamic recovery, non-constant growth rates, and topological information coming from the introduction of two HEMs. The proposed model has been able to provide quantitative predictions of the recrystallized fraction, the recrystallized grain size, and the flow stress curves for different conditions: a good indication that the basic mechanisms of recrystallization during and after deformation are well captured by the model. This simple model can therefore be considered as a tool for the optimization of thermomechanical processing at the industrial scale.

The method to determine the values of the parameters used by the model is also described. By obtaining and validating the parameters from different experimental data in different recrystallization regimes, parameter tuning becomes efficient. This also solves the problem of obtaining multiple solutions lacking a physical meaning. By using parameters obtained with such a methodology, the model is stable and its predictive capabilities are increased.

\section{Appendix A. List of symbols used}

The following notation is used in the article, some of the symbols were already used in [33].

\begin{tabular}{ll}
\hline$b$ & Burger's vector $(\mathrm{m})$ \\
$b_{g}$ & constant related to nucleation \\
$\tau$ & energy per unit dislocation length $(\mathrm{N})$ \\
$m$ & grain boundary mobility $\left(\mathrm{m}^{3} \mathrm{~s}^{-1} \mathrm{~N}^{-1}\right)$ \\
$d_{0}, D_{0}$ & initial average grain diameter $(\mathrm{m})$ \\
$d_{s s}$ & steady state grain size \\
$d_{d r x}$ & dynamically recrystallized grain size \\
$d_{p d r x}$ & post-dynamically recrystallized grain size \\
$D_{\max }$ & initial maximum grain diameter $(\mathrm{m})$ \\
$\bar{D}_{R X}$ & average grain diameter of recrystallized grains $(\mathrm{m})$
\end{tabular}

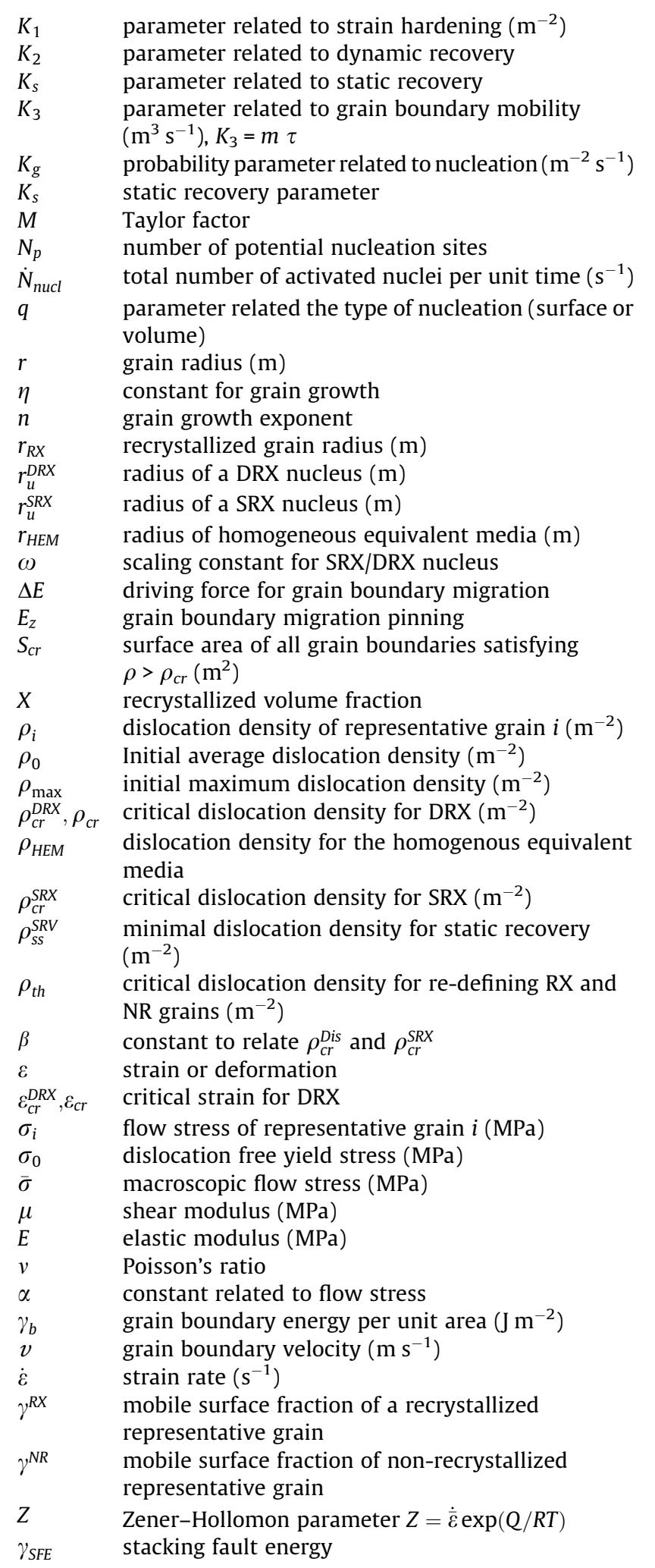

\section{Appendix B. Relative value for the fitting parameters}




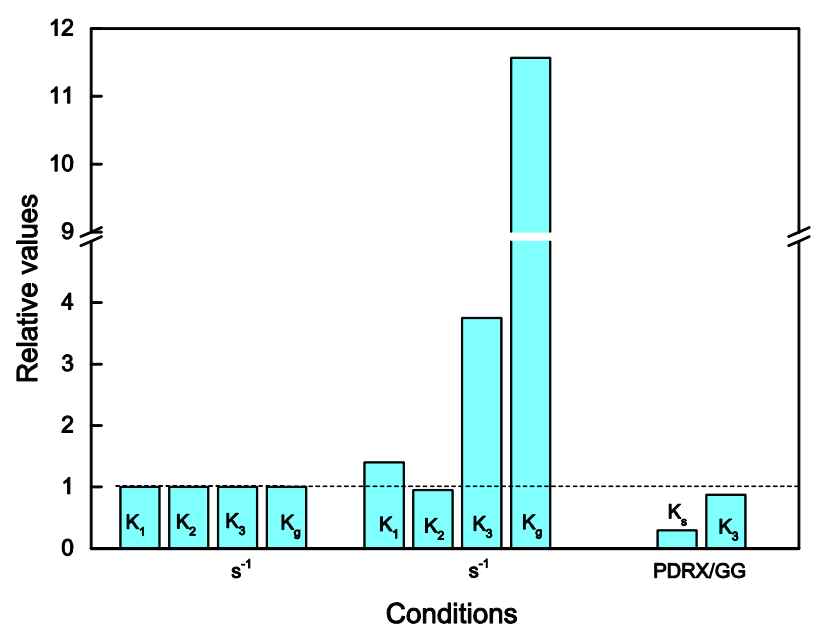

Fig. B-1. Relative evolution of $K$ parameters at $1000{ }^{\circ} \mathrm{C}$, with respect to the reference values obtained at $0.01 \mathrm{~s}^{-1}$ during DDRX.

\section{References}

[1] J.W. Christian, The Theory of Transformations in Metals and Alloys, third ed. Pergamon Press, Oxford; New York, 2002. pp. 851.

[2] F.J. Humphreys, M. Hatherly, Recrystallization and Related Annealing Phenomena, Second ed., Elsevier, Oxford, 2004.

[3] G.A. Wilber, J.R. Bell, J.H. Bucher, W.J. Childs, TMS-AIME 244 (1968) 23052308.

[4] A. Dehghan-Manshadi, The evolution of recrystallization during and following hot deformation. Ph.D thesis, Deaking University, Australia, 2007.

[5] R.A.P. Djaic, J.J. Jonas, J. Iron Steel Inst. 210 (1972) 256-261.

[6] R.A. Petkovic, M.J. Luton, J.J. Jonas, Acta Metall. 27 (1979) 1633-1648.

[7] M.J. Luton, R.A. Petkovic, J.J. Jonas, Acta Metall. 28 (1980) 729-743.

[8] Z. Xu, T. Sakai, Mater. Trans. JIM 32 (1991) 174-180.

[9] T. Sakai, M. Ohashi, K. Chiba, J.J. Jonas, Acta Mater. 36 (1988) 1781-1790.

[10] P.D. Hodgson, R.K. Gibbs, ISIJ Int. 32 (1992) 1329-1338.

[11] C. Roucoules, P.D. Hodgson, Mater. Sci. Technol. 11 (1995) 548-556.

[12] H. Beladi, P. Cizek, P.D. Hodgson, Acta Materialia 59 (2011) 1482-1492.

[13] N. Yazdipour, P.D. Hodgson, Computat. Mater. Sci. 54 (2012) 56-65.
[14] A. Dehghan-Manshadi, M.R. Barnett, P.D. Hodgson, Metall. Mater. Trans. A 39A (2008) 1359-1370.

[15] A. Dehghan-Manshadi, M.R. Barnett, P.D. Hodgson, Mater. Sci. Eng. A 485 (2008) 664-672.

[16] S. Cho, K. Kang, J.J. Jonas, ISIJ Int. 41 (2001) 63-69.

[17] S. Cho, K. Kang, J.J. Jonas, ISIJ Int. 41 (2001) 766-773.

[18] A. Kirihata, F. Siciliano, T.M. Maccagno, J.J. Jonas, ISIJ Int. 38 (1998) 187-195.

[19] S.H. Cho, Y.C. Yoo, J. Mater. Sci. 36 (2001) 4279-4284.

[20] S.H. Cho, Y.C. Yoo, J. Mater. Sci. 36 (2001) 4267-4272.

[21] D.S. Svyetlichnyy, ISIJ Int. 45 (2005) 1187-1193.

[22] B. Radhakrishnan, G.B. Sarma, T. Zacharia, Acta Materialia 46 (1998) 44154433.

[23] C. Roucoules et al., Mater. Sci. Eng. A 339 (2003) 1-9.

[24] R. Sandstrom, R. Lagneborg, Acta Metall. 23 (1975) 387-398.

[25] R. Sandstrom, R. Lagneborg, Acta Metall. 23 (1975) 481-488.

[26] G. Kugler, R. Turk, Acta Materialia 52 (2004) 4659-4668.

[27] H.S. Zurob, Y. Brechet, G. Purdy, Acta Materialia 49 (2001) 4183-4190.

[28] H.S. Zurob, C.R. Hutchinson, Y. Brechet, G. Purdy, Acta Materialia 50 (2002) 3077-3094.

[29] H.E. Vatne, E. Nes, Computat. Mater. Sci. 7 (1996) 5-10.

[30] H.E. Vatne, K. Marthinsen, R. Ørsund, E. Nes, Metall. Mater. Trans. A 27A (1996) 4133-4144.

[31] H.E. Vatne, F. Perocheau, H.E. Ekstrom, L. Poizat, E. Nes, J. Hagstrom, M. Lyttle, T. Furu, J. de Physique IV 11 (2001) 123-130.

[32] J. Hirsch, Virtual Fabrication of Aluminum Products: Microstructural Modeling in Industrial Aluminum Production, Wiley-VCH, 2007.

[33] P. Bernard, S. Bag, K. Huang, R.E. Logé, Mater. Sci. Eng. A 528 (2011) $7357-$ 7367.

[34] U.F. Kocks, J. Eng. Mater. Technol. 98 (1976) 76-85.

[35] H. Mecking, U.F. Kocks, Acta Metall. 29 (1981) 1865-1875.

[36] Y. Estrin, H. Mecking, Acta Metall. 32 (1984) 57-70.

[37] G.I. Taylor, Proc. R. Soc. London Ser. A 145 (1934) 362-387.

[38] M. Hillert, Acta Metall. 13 (1965) 227-238.

[39] F. Montheillet, O. Lurdos, G. Damamme, Acta Mater. 57 (2009) 1602-1612.

[40] W. Roberts, B. Ahlblom, Acta Metall. 26 (1978). 801-813-209.

[41] T. Sakai, M. Ohashi, Mater. Sci. Technol. 6 (1990) 1251-1257.

[42] X. Quelennec, E. Martin, L. Jiang, J.J. Jonas, J. Phys.: Confer. Series 240 (2010) 012082.

[43] T. Sakai, M. Ohashi, Mater. Sci. Forum 113-115 (1993) 521-526.

[44] T. Sakai, Static recovery, recrystallization and grain growth in dynamically recrystallized materials, Proceedings of ReX'96, the 3rd International Conference on Recrystallization and Related Phenomena, Edited by Terry R. McNelley, 1997, pp. 137-148.

[45] B. Derby, Acta Metall. Mater. 39 (1991) 955-962.

[46] A. Dehghan-Manshadi, J.J. Jones, P.D. Hodgson, M.R. Barnett, ISIJ Int. 48 (2008) 208-211. 